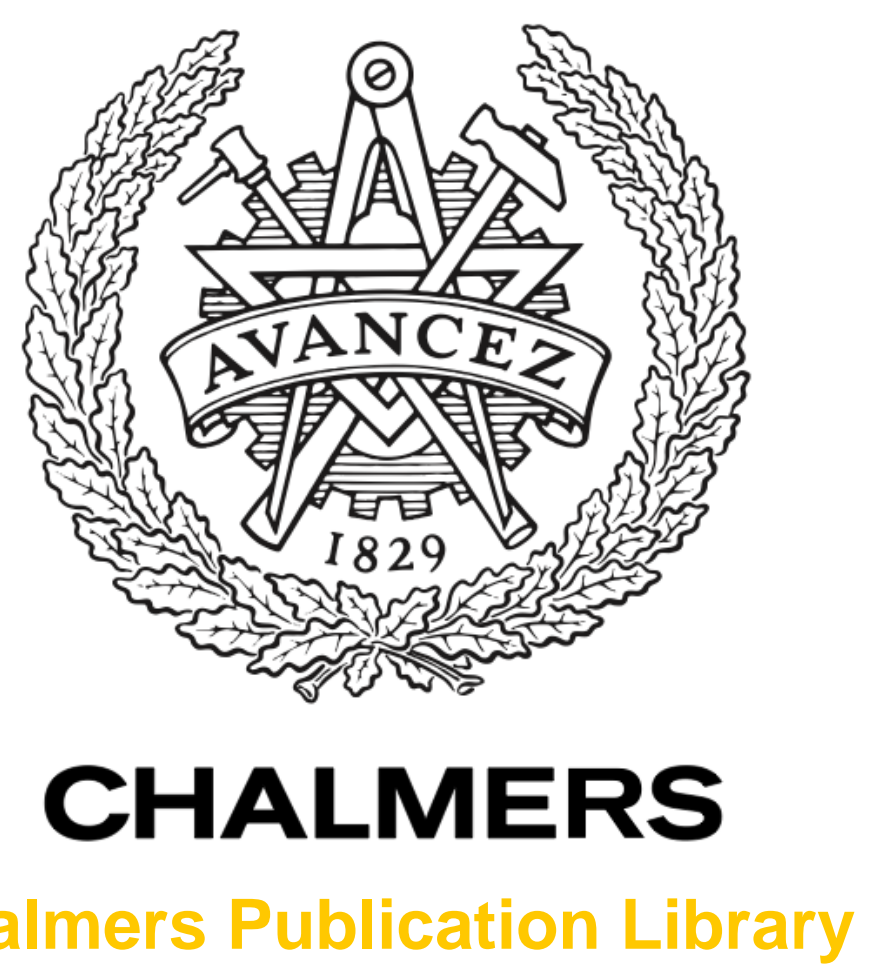

\title{
Phase coherence and energy relaxation in epitaxial graphene under microwave radiation
}

This document has been downloaded from Chalmers Publication Library (CPL). It is the author's version of a work that was accepted for publication in:

Applied Physics Letters (ISSN: 0003-6951)

Citation for the published paper:

Eless, V. ; Yager, T. ; Spasov, S. (2013) "Phase coherence and energy relaxation in epitaxial graphene under microwave radiation". Applied Physics Letters, vol. 103(9), pp. art. no.

093103.

http://dx.doi.org/10.1063/1.4819726

Downloaded from: http://publications.lib.chalmers.se/publication/185937

Notice: Changes introduced as a result of publishing processes such as copy-editing and formatting may not be reflected in this document. For a definitive version of this work, please refer to the published source. Please note that access to the published version might require a subscription. 


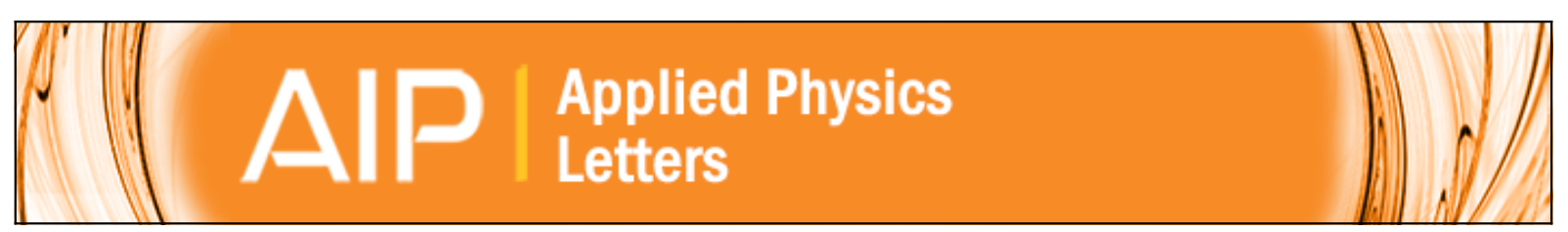

Phase coherence and energy relaxation in epitaxial graphene under microwave radiation

V. Eless, T. Yager, S. Spasov, S. Lara-Avila, R. Yakimova, S. Kubatkin, T. J. B. M. Janssen, A. Tzalenchuk, and V. Antonov

Citation: Applied Physics Letters 103, 093103 (2013); doi: 10.1063/1.4819726

View online: http://dx.doi.org/10.1063/1.4819726

View Table of Contents: http://scitation.aip.org/content/aip/journal/apl/103/9?ver=pdfcov

Published by the AIP Publishing

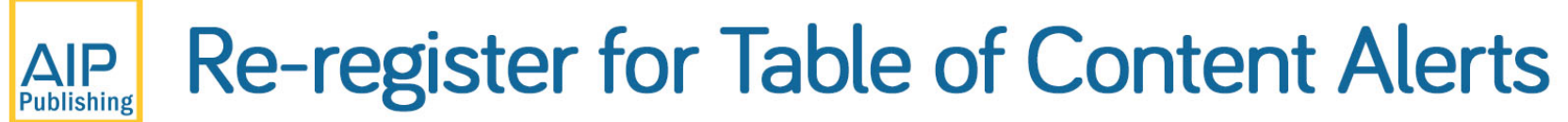




\title{
Phase coherence and energy relaxation in epitaxial graphene under microwave radiation
}

\author{
V. Eless, ${ }^{1,2, a)}$ T. Yager, ${ }^{3}$ S. Spasov, ${ }^{2}$ S. Lara-Avila, ${ }^{3}$ R. Yakimova, ${ }^{4,5}$ S. Kubatkin, ${ }^{3}$ \\ T. J. B. M. Janssen, ${ }^{1}$ A. Tzalenchuk, ${ }^{1,2}$ and V. Antonov ${ }^{2}$ \\ ${ }^{1}$ National Physical Laboratory, Hampton Road, Teddington TW11 0LW, United Kingdom \\ ${ }^{2}$ Physics Department, Royal Holloway, University of London, Egham, Surrey TW20 OEX, United Kingdom \\ ${ }^{3}$ Department of Microtechnology and Nanoscience (MC2), Chalmers University of Technology, \\ S-41296 Göteborg, Sweden \\ ${ }^{4}$ Department of Physics, Chemistry and Biology (IFM), Linköping University, S-58183 Linköping, Sweden \\ ${ }^{5}$ Graphensic AB, S-58330 Linköping, Sweden
}

(Received 26 June 2013; accepted 14 August 2013; published online 26 August 2013)

\begin{abstract}
We have performed low-temperature magnetotransport measurements on monolayer epitaxial graphene under microwave radiation and extracted the radiation-induced effective temperatures, energy relaxation, and the dephasing times. We established that the response of the graphene sample is entirely bolometric at least up to $170 \mathrm{GHz}$. Dynamic dephasing, i.e., the time-reversal symmetry breaking effect of the ac electromagnetic field rather than mediated by heating, may become significant in the terahertz frequency range and in samples with longer phase coherence time. [http://dx.doi.org/10.1063/1.4819726]
\end{abstract}

Carrier energy loss rates determine how the electrons cool down when heated above the lattice temperature. They influence thermal dissipation and heat management in graphene electronics ${ }^{1-3}$ as well as low-temperature applications such as quantum resistance metrology ${ }^{4,5}$ and hot-electron bolometers. ${ }^{6,7}$ Energy loss rates have previously been measured in exfoliated, CVD and epitaxial graphene grown on $\mathrm{SiC}$ using dc current heating of the electrons above the lattice temperature. ${ }^{8-11}$ At the same time, many of the proposed applications of graphene both at room- and at low temperatures involve operation at high frequencies. ${ }^{12,13}$ To evaluate the low-temperature performance of epitaxial graphene exposed to microwave radiation, we used quantum corrections to conductivity to find the electron temperature and determine the loss rates.

Quantum corrections to the charge transport in graphene have been extensively studied over the recent years. ${ }^{14-17}$ The corrections arise from the localization and interaction effects in the disordered Fermi liquid. The weak localisation (WL) correction to the conductivity is due to the coherence of electrons traversing the same trajectories in opposite directions. Experimentally, it manifests itself in negative magnetoresistance around the zero magnetic field. Any violation of the symmetry with respect to time reversal leads to dephasing. The symmetry breaking processes include all inelastic processes, e.g., scattering on electrons, phonons, or localised spins, dephasing due to static magnetic field or alternating electromagnetic field. The latter effect was studied theoretically $^{18}$ and to some extent experimentally ${ }^{19,20}$ in conventional 2DEGs, but not until now in graphene.

Interaction of microwave radiation with the electrons can, in principle, lead to two distinct effects, each contributing to the change in conductivity.

The absorption of radiation by the electron system leads to its heating to a temperature $T_{e}$ above the lattice temperature

a)vbe@npl.co.uk
$T_{L}$, governed by the energy relaxation time $\tau_{e}$. The increase in $T_{e}$ leads to the suppression of $\tau_{\phi}$ and consequently alters the WL correction to conductivity. In addition, the in-plane highfrequency electric field modifies the WL contribution to conductivity directly. Both the direct and indirect (through heating) effect of microwaves on WL increase with microwave power. Any direct suppression of quantum coherence in graphene due to microwave radiation (dynamic dephasing) has to be distinguished from a contribution from the heating effect of the electromagnetic field. Therefore, temperature dependence of WL correction to conductivity cannot a priori be used for the electron thermometry under high-frequency excitation. WL and electron-electron (EE) interaction corrections to the conductivity depend on the temperature in the same way, but they can be separated by suppressing WL in magnetic field. At low temperatures and once WL is suppressed, the conductivity only depends on temperature through the EE interaction, which can be used as an electron thermometer.

The investigated graphene samples were grown on the Si-terminated face of silicon carbide ( $\mathrm{SiC}(0001))$ by graphitisation at $2000{ }^{\circ} \mathrm{C}$ in $1 \mathrm{~atm}$ of argon. They are $n$-doped with carrier concentration and mobility of $n \approx 5 \times 10^{11} \mathrm{~cm}^{-2}$ and $\mu \approx 7.5 \times 10^{3} \mathrm{~cm}^{2} / \mathrm{V}$ s at $4 \mathrm{~K}$ correspondingly. Nine devices were fabricated on a $7 \times 7 \mathrm{~mm}^{2}$ chip. Two devices had a Hall bar geometry with eight electrical contacts, which allowed for four-terminal measurements of the longitudinal and Hall resistance. These devices were used in microwave measurements up to $10 \mathrm{GHz}$. Further nine devices were used in measurements at $170 \mathrm{GHz}$ and will be described later in the text. All devices showed similar behaviour with a parameter variation of $\approx 10 \%$. The whole chip was covered with a polymer bilayer (PMMA-MAA/ZEP520A) both for protection of graphene devices and to enable photochemical control of the charge carrier density in graphene by deep UV light. ${ }^{21}$

In a magnetic field, sufficient to completely suppress weak localisation, the influence of alternating electromagnetic fields on the conductivity reduces simply to the heating of the 
electron system and the residual temperature dependence is due to the EE interactions. In this regime, therefore we can relate the effective temperature of the electrons to the power of the alternating electromagnetic field by comparing the change in conductance (or resistance) due to power and due to the ambient temperature. Theoretically, WL is completely suppressed in the field $0.3-0.4 \mathrm{~T}$ at which the magnetic length $\left(l_{B}=\sqrt{\hbar / 2 e B}\right)$ becomes comparable to the mean free path in our samples $(l \approx 45-50 \mathrm{~nm})$. Experimentally, however, we found that the resistance is flat between 0.1 and $0.6 \mathrm{~T}$ and all analysis has been performed in the range $B \leq|0.2| \mathrm{T}$. Figure 1 illustrates the power-to-temperature calibration procedure for one representative set of data.

To quantify the influence of microwave radiation on quantum coherence, we plot the inverse phase coherence time $\tau_{\phi}^{-1}$ as a function of the (effective) temperature (Figure 2). The phase coherence time was determined by fitting the weak localisation peak to the theory. ${ }^{22}$ The zero field, temperature-dependent analytic correction due to localization effects in graphene reads

$$
\Delta \rho_{W L-F i t}(B=0, \bar{T})=\frac{e^{2} \rho^{2}}{\pi h}\left[\ln \left(1+2 \frac{\tau_{\phi}}{\tau_{i}}\right)-2 \ln \left(\frac{\tau_{\phi} / \tau_{t r}}{1+\tau_{i} / \tau_{*}}\right)\right],
$$

where the relaxation time is calculated as $\tau_{t r}=l / v_{F}=$ $h /\left(2 e^{2} \rho_{x x} v_{F} \sqrt{\pi n}\right) \approx 0.05 \mathrm{ps}$ and the scattering times $\tau_{\phi}, \tau_{i}$ (intervalley), $\tau_{*}$ (intravalley) are found by fitting the measured $\rho_{x x}(B, \bar{T})$ to Eq. (2)

$$
\frac{\Delta \rho(B, \bar{T})}{\rho^{2}}=-\frac{e^{2}}{\pi h}\left[F\left(\frac{B}{B_{\phi}}\right)-F\left(\frac{B}{B_{\phi}+2 B_{i}}\right)-2 F\left(\frac{B}{B_{\phi}+B_{*}}\right)\right]
$$

with $F(z)=\ln (z)+\Psi\left(\frac{1}{2}+\frac{1}{z}\right)$ and $\tau_{\phi, i, *}=\frac{\hbar}{4 D e} B_{\phi, i, *}^{-1}$.

The conclusion from Figure 2 is that the microwave radiation has exactly the same effect on quantum coherence as the change in the electron temperature. This means that the response is entirely bolometric in the whole parameter space studied and that the dynamic dephasing process is negligible.

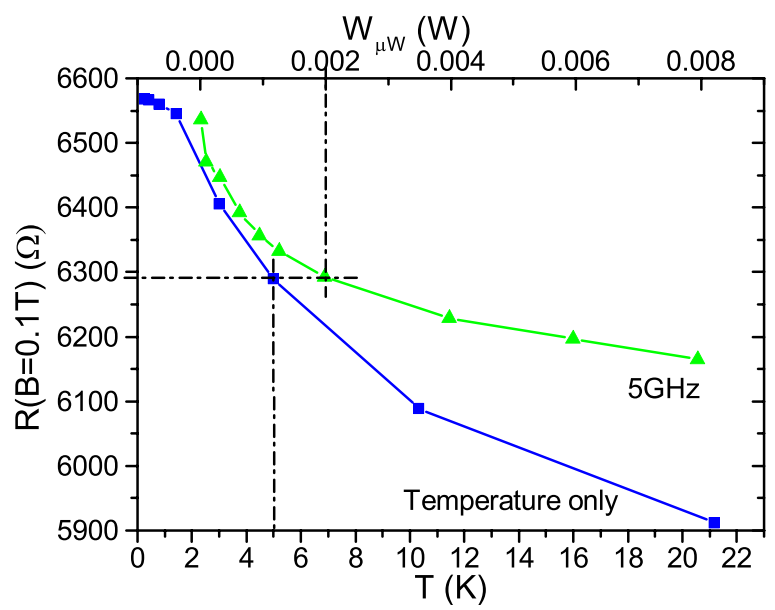

FIG. 1. Comparison between the effect of ambient temperature and microwave radiation on the sample resistance in a magnetic field suppressing WL. Dashed lines illustrate the $W_{\mu w}$-to- $T_{e}$ calibration.

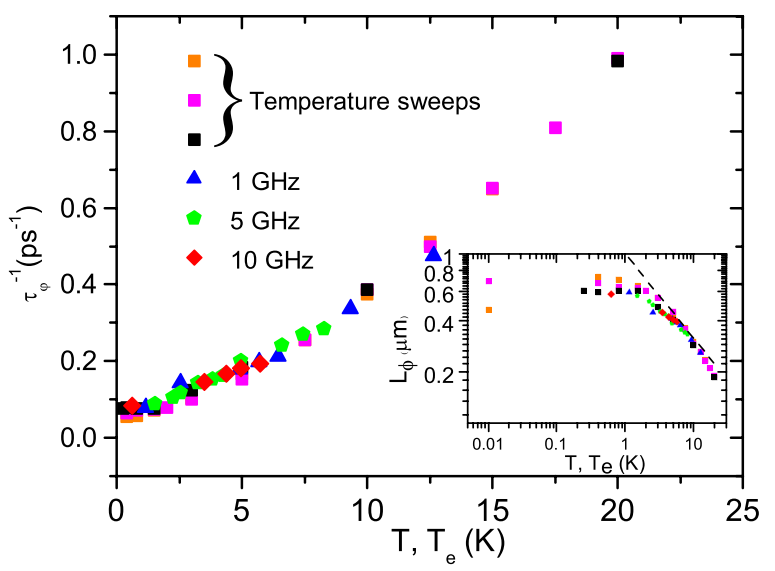

FIG. 2. Inverse phase coherence time and, inset, length as a function of the effective temperature.

The fastest scattering process determining the electron momentum relaxation rate is scattering on donors on the $\mathrm{SiC}$ surface, whereas $\tau_{i}$ and $\tau_{*}$ are much longer. We also note that the fits are not particularly sensitive to the values of $\tau_{i}$ and $\tau_{*}$, but qualitatively we conclude that they are virtually temperature-independent.

The values of the phase coherence length $L_{\phi}=\sqrt{D \tau_{\phi}}$, resulting from the fit to the WL theory are presented in Figure 2 inset. At low temperatures, $L_{\phi}$ reaches the micrometer range. The dashed line shows the fit to $T^{-1 / 2}$ in the appropriate temperature range.

The energy relaxation rate per carrier $P$ is determined as $P=\frac{W}{n A}$, where $W$ is the heating power, $\mathrm{n}$ - carrier density and A-area of the sample. The relaxation rate depends on the temperature of the electrons resulting from the heat, $T_{e}$ and the temperature of the lattice $T_{L}$. Above we have established the effective electron temperature of a graphene sample upon microwave excitation. Figure 3 shows the scaling of the temperature with microwave power, $W_{\mu w}$. The same heating effect is produced by dc power dissipation $W_{d c}$. In both cases, the power is $\propto\left(T_{e}^{3}-T_{L}^{3}\right)$ in agreement with our conclusion that microwave excitation of a graphene sample results only in heating. This scaling is consistent with the supercollision cooling mechanism ${ }^{23}$ and was previously observed at relatively high $T_{L}$ in experiments using dc current heating ${ }^{11,24}$ and optical excitation. ${ }^{25}$ Comparing the

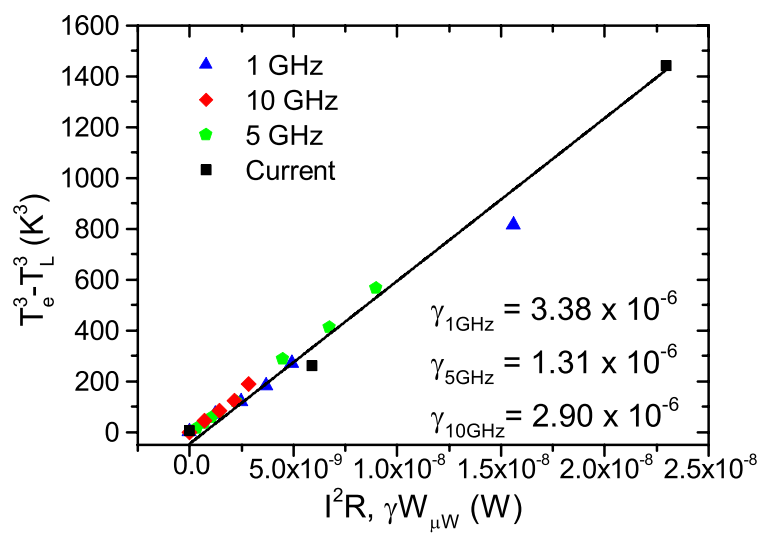

FIG. 3. Scaling of the electron temperature with the dissipated dc electric power and the microwave power at various frequencies. 


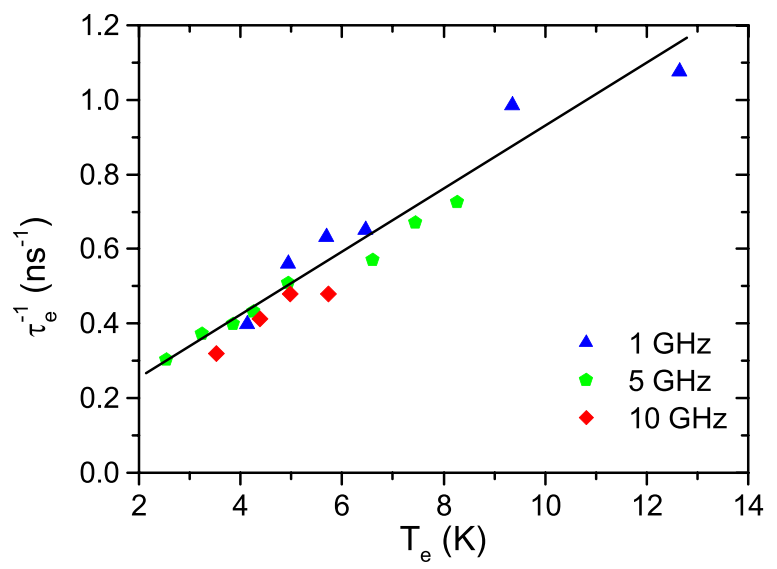

FIG. 4. Inverse energy relaxation time as a function of the effective temperature.

slopes, we determine both the frequency-dependent coupling coefficient $\gamma$ of the microwave power $W_{\mu w}$ to the sample and the energy relaxation rate. From $W_{d c}=I^{2} R=\alpha\left(T_{e}^{3}-T_{L}^{3}\right)$, we get $\alpha=5.38 \times 10^{-18} \mathrm{~W} \mathrm{~K}^{-3}$. Equating $W_{d c}=\gamma W_{\mu w}$ $=\alpha\left(T_{e}^{3}-T_{L}^{3}\right)$, we get $\gamma$ in the range of $10^{-6}$ slightly varying for different frequencies.

Further, we determine the energy relaxation time as $\tau_{e}=\frac{\pi^{2} k_{b}^{2} T_{e}^{2}}{3 E_{F} P}=\frac{\pi^{2} k_{b}^{2} T_{e}^{2}}{3 E_{F} \alpha\left(T_{e}^{3}-T_{L}^{3}\right)}$ for different frequencies and microwave powers, Figure 4. As expected for high electron overheating against the lattice, $T_{e} \gg T_{L}, \tau_{e} \propto 1 / T_{e}$.

Using frequencies of 1 to $10 \mathrm{GHz}$, we have found $\tau_{e}>1 \mathrm{~ns}$. From Figure 2, we obtain $\tau_{\phi}$ in the range $1-15 \mathrm{ps}$. Thus for all microwave powers, we find that $\tau_{e} \gg \tau_{\phi}$. We can now compare the experimentally established characteristic times with the period of microwave radiation $t . t=2 \pi / f$ is in the range between 0.6 and $7 \mathrm{~ns}$ and therefore $\tau_{\phi} \ll t \approx \tau_{e}$. So, while there is enough time to establish the temperature in the electron system over the period of microwave radiation, there is not enough time to induce the dynamic dephasing. The latter process may become significant at frequencies in the terahertz range.

To probe the response of the graphene sample to electromagnetic radiation at much higher frequencies, we used a quasioptical scheme shown in Figure 5. The graphene sample was fitted with a log-periodic antenna to enhance coupling (Fig. 5(a)). The sample thermalised at the cold stage of an optical cryostat was illuminated by a Gunn diode emitting at $170 \mathrm{GHz}$ through a series of filtered windows installed at
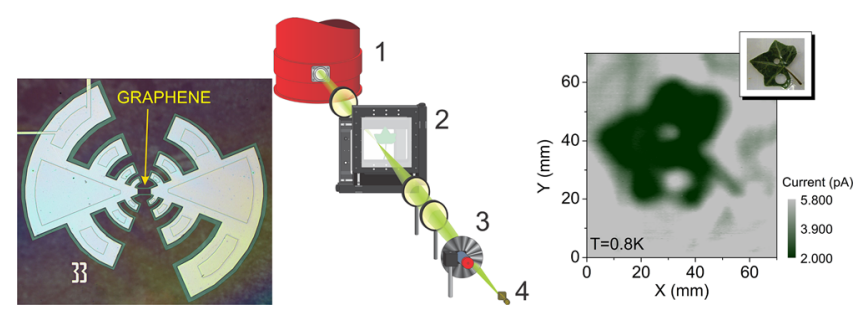

FIG. 5. (a) Optical micrograph of a $100 \times 60 \mu \mathrm{m}^{2}$ graphene strip placed in the centre of a log-periodic antennae; (b) Measurement setup: (1) refrigerator with the graphene sensor, (2) XY translation stage, (3) mechanical chopper, (4) Gunn diode source; (c) False-colour image of an ivy leaf illuminated by $170 \mathrm{GHz}$ radiation from the Gunn diode and detected by a voltage biased $(1 \mathrm{mV})$ graphene device held at $\mathrm{T}=0.8 \mathrm{~K}$; Inset: optical image of the same leaf.

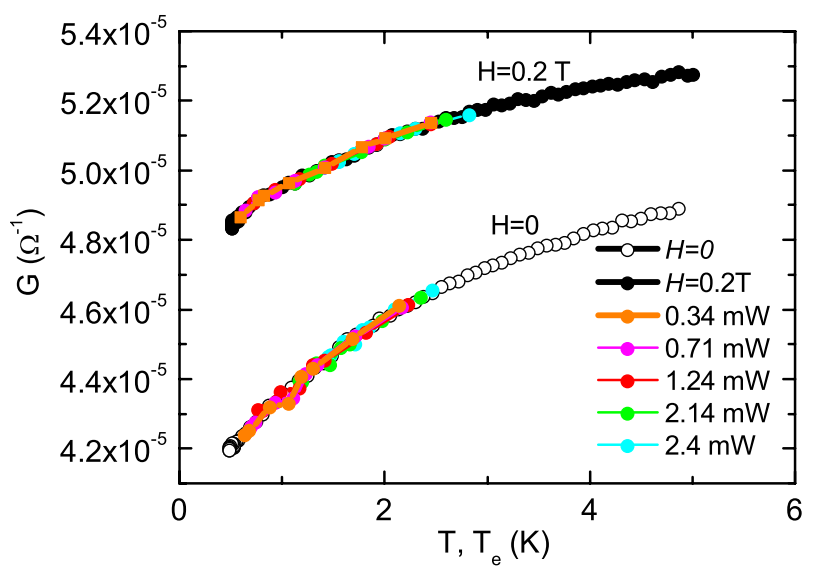

FIG. 6. Response of a graphene device in Figure 5(a) to $170 \mathrm{GHz}$ radiation.

different stages inside the cryostat (Fig. 5(b)). A graphic illustration of the sample response to the incident radiation is shown in Fig. 5(c): an ivy leaf was raster-scanned under the beam and the sample response was recorded as a function of the leaf position. The leaf attenuates the radiation power incident on the graphene sample.

In Figure 6, we show that the sample response to even $170 \mathrm{GHz}$ radiation is entirely bolometric. As before we determined the radiation-induced effective electron temperature by suppressing the weak localisation peak in magnetic field and comparing the conductance change associated with the sub-terahertz radiation with that due to change in the ambient temperature. We then adopted a simplified analytical approach, whereby we compare the change in the amplitude of the weak localisation peak at any given radiation-induced effective electron temperature and the corresponding ambient temperature, rather than the full $\tau_{\phi}$ analysis. Both approaches give similar results. We see that the amplitude of the weak localisation correction to conductivity is again entirely determined by the temperature of the electrons.

In summary, we have shown that the response to microwave radiation of an epitaxial graphene sample produced on $\mathrm{SiC}$ (0001) is entirely bolometric, i.e., due to heating of the electron gas, up to at least $170 \mathrm{GHz}$ and established the energy relaxation and the phase coherence times from microwave measurements.

Direct dephasing process may become important at $\mathrm{THz}$ frequencies and in graphene samples with longer phase coherence. In monolayer graphene on $\mathrm{SiC}(0001) \tau_{\phi}$ is limited by spin-flip scattering at low temperatures. The spin-flip process can be suppressed by in-plane magnetic field. ${ }^{26,27}$ Alternatively, for observation of the dynamic dephasing, one can use multilayer graphene on $\mathrm{SiC}(000 \overline{1})$.

The authors thank Harriet van der Vliet, Stephen Giblin, and Ruth Pearce for help with experiments and V. Fal'ko for useful discussions. This work was supported by the Swedish Research Council and Foundation for Strategic Research, EPSRC Grant EP/K016822/1 and EU FP7 STREP ConceptGraphene.

${ }^{1}$ A. A. Balandin, Nature Mater. 10, 569-581 (2011).

${ }^{2}$ K. M. Shahil and A. A. Balandin, Solid State Commun. 152, 1331-1340 (2012). 
${ }^{3}$ D. L. Nika and A. A. Balandin, J. Phys.: Condens. Matter 24, 233203 (2012).

${ }^{4}$ A. Tzalenchuk, S. Lara-Avila, A. Kalaboukhov, S. Paolillo, M. Syvajarvi, R. Yakimova, O. Kazakova, T. J. B. M. Janssen, V. Fal'ko, and S. Kubatkin, Nat. Nanotechnol. 5, 186-189 (2010).

${ }^{5}$ T. J. B. M. Janssen, N. E. Fletcher, R. Goebel, J. M. Williams, A. Tzalenchuk, R. Yakimova, S. Kubatkin, S. Lara-Avila, and V. I. Falko, New J. Phys. 13, 093026 (2011).

${ }^{6}$ J. Yan, M. H. Kim, J. A. Elle, A. B. Sushkov, G. S. Jenkins, H. M. Milchberg, M. S. Fuhrer, and H. D. Drew, Nat. Nanotechnol. 7, 472-478 (2012).

${ }^{7}$ K. C. Fong and K. C. Schwab, Phys. Rev. X 2, 031006 (2012).

${ }^{8}$ Z. Tan, C. Tan, L. Ma, G. T. Liu, L. Lu, and C. L. Yang, Phys. Rev. B 84, 115429 (2011).

${ }^{9}$ A. C. Betz, F. Vialla, D. Brunel, C. Voisin, M. Picher, A. Cavanna, A. Madouri, G. Fève, J. M. Berroir, B. Plaçais, and E. Pallecchi, Phys. Rev. Lett. 109, 056805 (2012).

${ }^{10}$ A. M. R. Baker, J. A. Alexander-Webber, T. Altebaeumer, and R. J. Nicholas, Phys. Rev. B 85, 115403 (2012).

${ }^{11}$ A. M. R. Baker, J. A. Alexander-Webber, T. Altebaeumer, S. D. McMullan, T. J. B. M. Janssen, A. Tzalenchuk, S. Lara-Avila, S. Kubatkin, R. Yakimova, C. T. Lin, L. J. Li, and R. J. Nicholas, Phys. Rev. B 87, 045414 (2013).

${ }^{12}$ Y.-M. Lin, C. Dimitrakopoulos, K. A. Jenkins, D. B. Farmer, H.-Y. Chiu, A. Grill, and P. Avouris, Science 327, 662 (2010).

${ }^{13}$ Y.-M. Lin, A. Valdes-Garcia, S.-J. Han, D. B. Farmer, I. Meric, Y. Sun, Y. Wu, C. Dimitrakopoulos, A. Grill, P. Avouris, and K. A. Jenkins, Science 332, 1294-1297 (2011).
${ }^{14}$ F. V. Tikhonenko, D. W. Horsell, R. V. Gorbachev, and A. K. Savchenko, Phys. Rev. Lett. 100, 056802 (2008).

${ }^{15}$ F. V. Tikhonenko, A. A. Kozikov, A. K. Savchenko, and R. V. Gorbachev, Phys. Rev. Lett. 103, 226801 (2009).

${ }^{16}$ S. Lara-Avila, A. Tzalenchuk, S. Kubatkin, R. Yakimova, T. J. B. M. Janssen, K. Cedergren, T. Bergsten, and V. Fal'ko, Phys. Rev. Lett. 107, 166602 (2011).

${ }^{17}$ J. Jobst, D. Waldmann, I. V. Gornyi, A. D. Mirlin, and H. B. Weber, Phys. Rev. Lett. 108, 106601 (2012).

${ }^{18}$ V. I. Fal'ko, Sov. Phys. JETP 65, 397-399 (1987).

${ }^{19}$ S. A. Vitkalov, G. M. Gusev, Z. D. Kvon, and G. I. Leviev, JETP Lett. 43, 185-188 (1986).

${ }^{20}$ S. A. Vitkalov, G. M. Gusev, Z. D. Kvon, G. I. Leviev, and V. I. Fal'ko, Sov. Phys. JETP 67, 1080-1084 (1988).

${ }^{21}$ S. Lara-Avila, K. Moth-Poulsen, R. Yakimova, T. Bjornholm, V. Fal'ko, A. Tzalenchuk, and S. Kubatkin, Adv. Mater. 23, 878 (2011).

${ }^{22}$ E. McCann, K. Kechedzhi, V. I. Fal'ko, H. Suzuura, T. Ando, and B. L. Altshuler, Phys. Rev. Lett. 97, 146805 (2006).

${ }^{23}$ J. C. W. Song, M. Y. Reizer, and L. S. Levitov, Phys. Rev. Lett. 109, 106602 (2012).

${ }^{24}$ A. C. Betz, S. H. Jhang, E. Pallecchi, R. Ferreira, G. Feve, J. M. Berroir, and B. Placais, Nat. Phys. 9, 109-112 (2013).

${ }^{25}$ M. W. Graham, S.-F. Shi, D. C. Ralph, J. Park, and P. L. McEuen, Nat. Phys. 9, 103-108 (2013).

${ }^{26}$ E. McCann and V. I. Fal'ko, Phys. Rev. Lett. 108, 166606 (2012).

${ }^{27}$ M. B. Lundeberg, R. Yang, J. Renard, and J. A. Folk, Phys. Rev. Lett. 110, 156601 (2013). 